\title{
Rationale, Mission and Vision for a National Centre of Affective Disorders in Germany
}

\author{
Authors \\ Andreas Reif1', Bernhard T. Baune ${ }^{2}$, Jürgen Deckert ${ }^{3}$, Georg Juckel ${ }^{4}$, Sarah Kittel-Schneider ${ }^{3}$, Tilo Kircher ${ }^{5}$, Johannes \\ Kornhuber $^{6}$, Rainer Rupprecht ${ }^{7}$, Michael Bauer ${ }^{8}$
}

\section{Affiliations}

1 Department of Psychiatry, Psychosomatic Medicine and Psychotherapy, University Hospital Frankfurt - Goethe University, Frankfurt am Main, Germany

2 Department of Psychiatry, University Hospital Münster, University of Münster, Münster, Germany

3 Department of Psychiatry, Psychosomatics and Psychotherapy, Center of Mental Health, University Hospital of Würzburg, Würzburg, Germany

4 Department of Psychiatry, Psychotherapy and Preventive Medicine, LWL University Hospital, Ruhr University Bochum, Germany

5 Department of Psychiatry and Psychotherapy, PhilippsUniversity Marburg, Marburg, Germany

6 Department of Psychiatry and Psychotherapy, University Hospital Erlangen, Friedrich-Alexander-University of Erlangen-Nuremberg, Germany

7 Department of Psychiatry and Psychotherapy, University Regensburg, Regensburg, Germany

8 Department of Psychiatry and Psychotherapy, Medical Faculty, Carl Gustav Carus University Hospital, Technische Universität Dresden, Dresden, Germany

\section{Key words}

affective disorders, bipolar disorder, major depression, anxiety, network

received $\quad 13.10 .2021$

revised 13.10 .2021

accepted 05.11.2021

published online 17.12 .2021

\section{Bibliography}

Pharmacopsychiatry 2022; 55: 65-72

DOI 10.1055/a-1697-5854

ISSN $0176-3679$

(C) 2021. Thieme. All rights reserved.

Georg Thieme Verlag, Rüdigerstraße 14,

70469 Stuttgart, Germany

\section{Correspondence}

Prof. Dr. Andreas Reif

Department of Psychiatry, Psychosomatic Medicine and

Psychotherapy

University Hospital Frankfurt - Goethe University

Frankfurt am Main

Germany

andreas.reif@kgu.de

\section{ABSTRACT}

Affective disorders are common, complex disorders representing one of the major challenges to global health in the 21st century. To mitigate the burden of disease, substantial public health efforts need to be undertaken since research on the causes and adequate treatment requires multidisciplinary approaches. These should integrate translational, and clinical research, aided by technological advancements in collecting and analysing comprehensive data. Here we present the rationale, concept, mission and vision of the recently founded $\mathrm{Na}$ tional Centre of Affective Disorders (NCAD) in Germany. NCAD founding partners build on their previous successful cooperation within the German Research Network for Mental Disorders funded by the Federal Ministry of Education and Research (BMBF). They form an internationally pre-eminent network of integrative excellence, leading in science and contributing significantly to the improved care of affective disorder patients. The partners will provide complementary structures and innovative methods across the entire translational continuum from bench to clinical and real-world settings. The vision of the NCAD is to foster cross-disciplinary research from basic neuroscience to public mental health by close translational collaboration between academia, non-university research institutions, and international partners, including industry, to deliver cutting-edge research, innovative clinical services and evidencebased training to young clinicians and scientists. The mission is to accomplish research in a highly translational manner, especially with respect to clinical studies in a trans-sectoral way. This approach aims to ensure continuous improvement in the treatment and care provided to patients and an interdisciplinary environment for high-level research and education in affective disorders. 


\section{Introduction}

Affective disorders (AD) are common complex disorders that cause an increasing burden on individuals and their families. They represent one of the greatest challenges to global health in the 21 st century and impose substantial economic costs to societies due to direct health care costs and productivity losses [1-5]. Common misdiagnoses, lack of, or insufficient access to treatment are leading causes for inappropriate or delayed intervention with devastating consequences such as worse functional outcome, elevated risk of suicide, and inferior response to biological and psychological interventions [6, 7]. To mitigate the burden of these disorders, substantial public health efforts need to be undertaken because research on the causes and adequate treatment requires multidisciplinary approaches, integrating basic brain and life sciences into clinical research, aided by technological advancements in collecting and analysing comprehensive data.

\section{Burden of disease}

The group of affective disorders mainly comprise the severe mental illnesses (SMI) major depressive disorder (MDD) and bipolar disorder (BD) as core diagnoses, that extend to other disorders, such as schizoaffective disorder and milder, sub-threshold disorders such as dysthymia. Moreover, anxiety disorders (ANX) should be addressed within the affective disorder spectrum for shared pathophysiology, risk constellations, and comorbidity.

Together, they are amongst the most common diseases worldwide, major depressive disorder being the leading cause of disability and affecting ca. 5 million patients every year in Germany alone. Accordingly, MDD is the most important single disorder with respect to inpatient healthcare cost and the second most important disorder with respect to sick leave days (depression accounting for 114 sick leave days per 100 person-years in Germany; https://www. dak.de/dak/download/gesundheitsreport-2017-2108948.pdf). While MDD has a lifetime prevalence of approx. $25 \%$ in females, and $12 \%$ in males [4], BD has a sex-balanced population lifetime prevalence of around 1-2\% [8]. Suicide, the most detrimental outcome of affective disorders, is the second most frequent cause of death in the age group of 15 to 45 years; in Germany, annually approximately 10,000 people die because of suicide. Despite huge efforts, this number remained stable during the last 15 years in contrast to a steady decline during the previous decades ( $\vee$ Fig. 1) highlighting the need for novel and more efforts to lower this toll of affective disorders. Also, life expectancy is significantly decreased in MDD and BD by about 10 years, even if premature death due to suicide is accounted for $[9,10]$. Moreover, parental affective disorders and anxiety disorders in the perinatal period are common, with $10-15 \%$ of mothers and about $5 \%$ of fathers being affected, resulting in negative effects not only in the parents but also in the development of the exposed children [11-13]. This calls for preventive measures in this vulnerable group. These numbers illustrate the huge importance of affective disorders regarding socio-economic costs, years of life lost due to disability, mortality, and, more importantly, individual suffering of patients and their relatives.

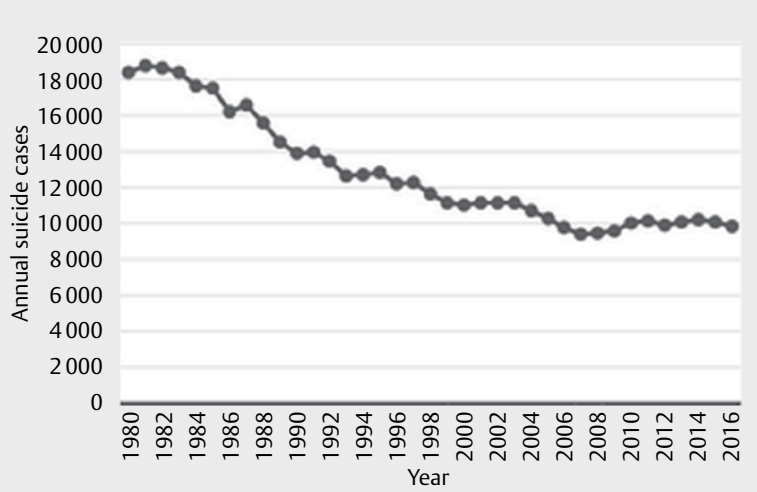

- Fig. 1 Absolute number of annual suicide cases in Germany (Source: destatis.de [2]).

\section{Unmet needs in affective disorders: the rationale for coordinated efforts}

Neuroscience has made tremendous progress in the last 50 years to uncover the functioning of neurons and their circuits on the micro- and meso-scale. But this progress has yet to be translated into advances within the field of mental disorders, which holds true for diagnostic as well as all therapeutic pillars. Diagnoses of mental disorders still rely on operationalized, "atheoretical" diagnostic systems such as Diagnostic and Statistical Manual of mental disorders (DSM) and International Classification of Disease (ICD): whatever iteration is looked at, diagnoses (that did not substantially change in the field of affective disorders during the last 40 years) are made upon checking and adding individual symptoms, which are explicitly not mapped onto biological mechanisms. Affective disorders especially are considered as a highly heterogeneous group of disorders, encompassing a variety of (neuro)-biological mechanisms, complex genetics, and a plethora of environmental influences [14-16]. Given this complexity, any attempt to uncover disease mechanisms by simply using DSM or ICD criteria is therefore doomed to fail. This especially speaks to the face validity of animal models; given that e. g., MDD is a heterogeneous group of disorders, there is no simple animal model of MDD. Importantly, almost all of the animal models studied feature trait-like abnormalities while affective disorders are prototypically episodic, i. e., they are state-like disorders. As a consequence of the flawed symptombased approach to uncover disease mechanisms (by studying DSMdefined phenotypes), the pathophysiology of the most common disorder - or rather, group of disorders - worldwide remains elusive. We are left with numerous proposed pathomechanisms that have not been convincingly unified or assigned to a given patient at the individual level. Therefore, we are stuck with decade-old treatments. With respect to psychopharmacological therapy, antidepressants that primarily target the monoaminergic transmitter system were developed 70 years ago and thereafter, only have been refined [17]. Likewise, lithium, the first-line prophylaxis therapy for BD in most guidelines, has been in use for many decades now, with no other mood stabilizer as effective [18]. Recently, esketamine was introduced in the treatment of MDD [19], and some psychedelic compounds are in late clinical development, such 
as psilocybin [20]; yet again, these compounds were synthesized a century ago and extensively been tested in the 1960s. The most effective therapy in (especially treatment-resistant) MDD, namely electro-convulsive therapy (ECT), has been developed in the 1930s and is still commonly used; other neuro-stimulatory treatments such as repetitive transcranial magnetic stimulation (rTMS) and transcranial direct current stimulation (tDCS) have failed to demonstrate equal efficacy [21-23]. Finally, cognitive-behavioural therapy (CBT), the best evaluated and most frequently used psychotherapy method used in AD, dates back to the 1960 s as well. The so-called "third wave of psychotherapy" incorporates approaches such as mindfulness and radical acceptance, which follow philosophical concepts that can be traced back thousands of years but as well do not target specific neural mechanisms [24,25]. There is a striking discrepancy between the prevalence and clinical relevance of affective disorders and the apparent lack of novel and better treatments, especially regarding treatment-resistant patients or with respect to the safety profile.

\section{The case for precision medicine: identifying mechanisms is essential for targeted treatment}

In recent years, the approach of "precision medicine" has gained increasing attention, mainly driven by progress in oncology and immune-mediated disorders. Precision medicine is "a medical model that proposes the customization of healthcare, with medical decisions, treatments, practices, or products being tailored to the individual patient, instead of a one-drug-fits-all model. In precision medicine, diagnostic testing is often employed for selecting appropriate and optimal therapies based on the context of a patient's genetic content or other molecular or cellular analysis" $[26,27]$. This directly points to the necessity of Predictive Markers (PM) that can govern the choice for the right treatment and predictors for the course of illness as well as therapy response. Such PM could comprise genetics, peripheral fluid biomarkers, brain imaging from several modalities, and objective behavioural assessment by using digital tools such as smartphone-sensor combinations (ecological momentary assessment, EMA: “digital phenotyping") to name but a few. However, such PMs crucially depend on the knowledge of underlying disease mechanisms, which are flagged by the respective marker (set), and respond to targeted treatments. As laid out above, such disease mechanisms cannot be identified if DSM- or ICD-diagnoses are exclusively used as defining criteria, as many pathomechanisms converge to an affective disorders syndrome. Rather, more fundamental biologically defined phenotypes should be taken as a starting point. The Research Domain Criteria initiative, proposed a few years ago [28], was the first step in this direction but has not yet been stringently applied to generate novel treatments. An alternative scenario is to begin with one of the proposed pathomechanisms of affective disorders, develop PM based thereon (which will likely cut across current diagnostic boundaries) and design fitting treatment studies. For instance, assuming that low-grade inflammatory processes underly some forms of affective disorders, anti-inflammatory compounds have repeatedly been tested for their efficacy in MDD and BD, with mixed outcomes [29, 30]: this is not surprising given that mainly DSM criteria were applied for study intake, but not an inflammatory biomarker profile as it should have been done following the logic of precision medicine. To conduct such studies, large patient numbers are needed both to filter out those displayed by a pathological biomarker profile, as well as institutions that are able to apply a wide variety of biomarker tests in a clinical research setting.

\section{Understanding and addressing the trajectories of early-onset disorders}

$A D$ result from complex vulnerability and risk factor constellations including familial, genetic and environmental (e. g., perinatal, early adversity, and life events) as well as individual neurobiological factors [31, 32]. They often evolve from unspecific symptoms as well as preceding syndromes and conditions (such as anxiety, attentiondeficit hyperactivity disorder (ADHD), affective dysregulation, and substance use) or evolve from an asymptomatic at-risk stage to the emergence of prodromal (affective) symptoms in adolescence or early adulthood, leading to the first episode of MDD or mania, and eventually to an unpredictable, relapsing course with poor psychosocial functioning throughout the lifespan [33, 34]. The descriptive developmental trajectories of emerging psychopathology and antecedent disorders have been identified from prospective-longitudinal population-representative or offspring studies. However, the exact underlying mechanisms of symptom progression remain far from conclusive. Furthermore, there are currently no models linking symptom development and outcome with neurobiological underpinnings throughout adolescence and early adulthood. This may at least partly be because, during the past decades, clinical and basic research increasingly began to disperse with researchers working mainly in either field, which reduced communication between basic and clinical scientists. The development of sophisticated techniques, and the increasing number of complex technologies and data analyses, resulted in the specialisation of researchers and fragmentation into different research disciplines. Since findings from single disciplines are difficult to integrate into multidisciplinary, translational frameworks, there is an urgent need for educating a new generation of specifically trained clinician-scientists that are able to overcome this fragmentation and turn research into balanced translational efforts. Also, the establishment of a translational research net- and frameworks of sufficient size and capabilities is necessary to address these knowledge gaps.

Many cases of affective disorders are precursors earlier in life. These include e.g., ANX or ADHD [35,36]. The latter is the most common neurodevelopmental disorder, with a worldwide prevalence between 5 and $8 \%$ in children and $3 \%$ in adults, and characterized by a high rate of comorbid somatic and mental disorders [37]. ANX involves separation anxiety, selective mutism, and social anxiety disorder starting in childhood and adolescence, and panic disorder/agoraphobia and generalized anxiety disorder beginning in early adulthood. At an annual prevalence of $14 \%$, they are the most common group of mental disorders [38]. The comorbidity rate of ANX and ADHD with affective disorders is about $50 \%$ up to $70 \%[39,40]$. These early-onset disorders are, therefore, entry points into a negative mental health trajectory, and prevention, early detection, and treatment of ADHD and ANX may impede the later development of affective disorders and their detrimental consequences. Importantly, this opens opportunities for early primary, secondary, and tertiary prevention if the sequence of ADHD/ ANX to affective disorders is considered as a developmental process 
rather than mere comorbidity of distinct phenotypes. Such a developmental staging model conceptualizes a patient journey beginning with being "at-risk" (either genetically, or environmentally, or both), then developing unspecific and common sub-threshold symptoms in early childhood to further continue in neuro-psychodevelopmental disorders. Later, affective disorders add to the disease burden which can progress into severe, chronic, and treatment-resistant forms of the disease, suicide being the most fatal outcome [41]. Implementing early detection (using PM) and precisely timed preventive measures against disease progression and harnessing resilience against various forms of environmental insults will, therefore, have a huge and lasting impact on mental health at the population level [42]. Targeted, escalated treatments in later stages will prevent more severe, chronic, or even lethal trajectories, as illustrated in - Fig. 2, which charts neuronal and brain development against the disease trajectory of a fictitious patient from being at-risk to suffering from chronic affective disorders [43]. Examples of such secondary and tertiary prevention are interventions to prevent depression recurrence and, therefore, suicide [44]. Especially in primary, but also secondary prevention, resourcefriendly digital health interventions can address the huge unmet need in these prevalent disorders, while Predictive Markers can direct more complex therapies (novel and selective drugs, non-invasive brain stimulation) to more severely ill patients. Moreover, there is enormous progress integrating novel therapeutic approaches such as virtual reality and App-based interventions onto modern psychotherapy concepts [45-48].

The question as to how environmental risk factors interact with biological subtypes of these early-onset disorders to result in disease progression calls for longitudinal studies incorporating multi- modal data. Given the huge impact on individual suffering, socioeconomic cost, and the incapacitating effect of $A D$, prevention of disease progression however has an enormous effect on general mental health and enables a large number of affected patients to unleash their full potential, therefore justifying the effort to conduct longitudinal studies.

\section{The National Centre for Affective Disorders (NCAD): mission and vision}

To reach the respective goals, large and longitudinally studied cohorts and intervention trials are needed to chart affective disorders over the lifespan, appreciating their heterogeneity and applying a precision medicine model, which is needed to develop more targeted and more effective treatments. Such a demanding task calls for collaboration, as no single site will be able to recruit sufficiently sized samples on its own. In addition, mechanistic heterogeneity calls for in-depth knowledge, expertise and methodological capabilities that require experts from many fields. The previous successful cooperation within the consortia BipoLife [49-51], OptiMD and ProtectAD [52], funded by the German Ministry of Research and Education (BMBF), and the DFG funded networks FOR2107 [53], is an excellent starting point to build the National Centre for Affective Disorders (NCAD) in Germany. The vision of NCAD is to reduce the burden imposed by $A D s$ via targeted prevention and treatments. To do so, its primary aim is to foster research in affective disorders with special emphasis on MDD, BD, treatment-resistant depression, suicidality and ANX. The full severity and chronicity spectrum from milder forms of the disease, mainly seen in primary care, to severe and chronic courses, is covered by these approaches. NCAD is complemented by graduate schools and re-

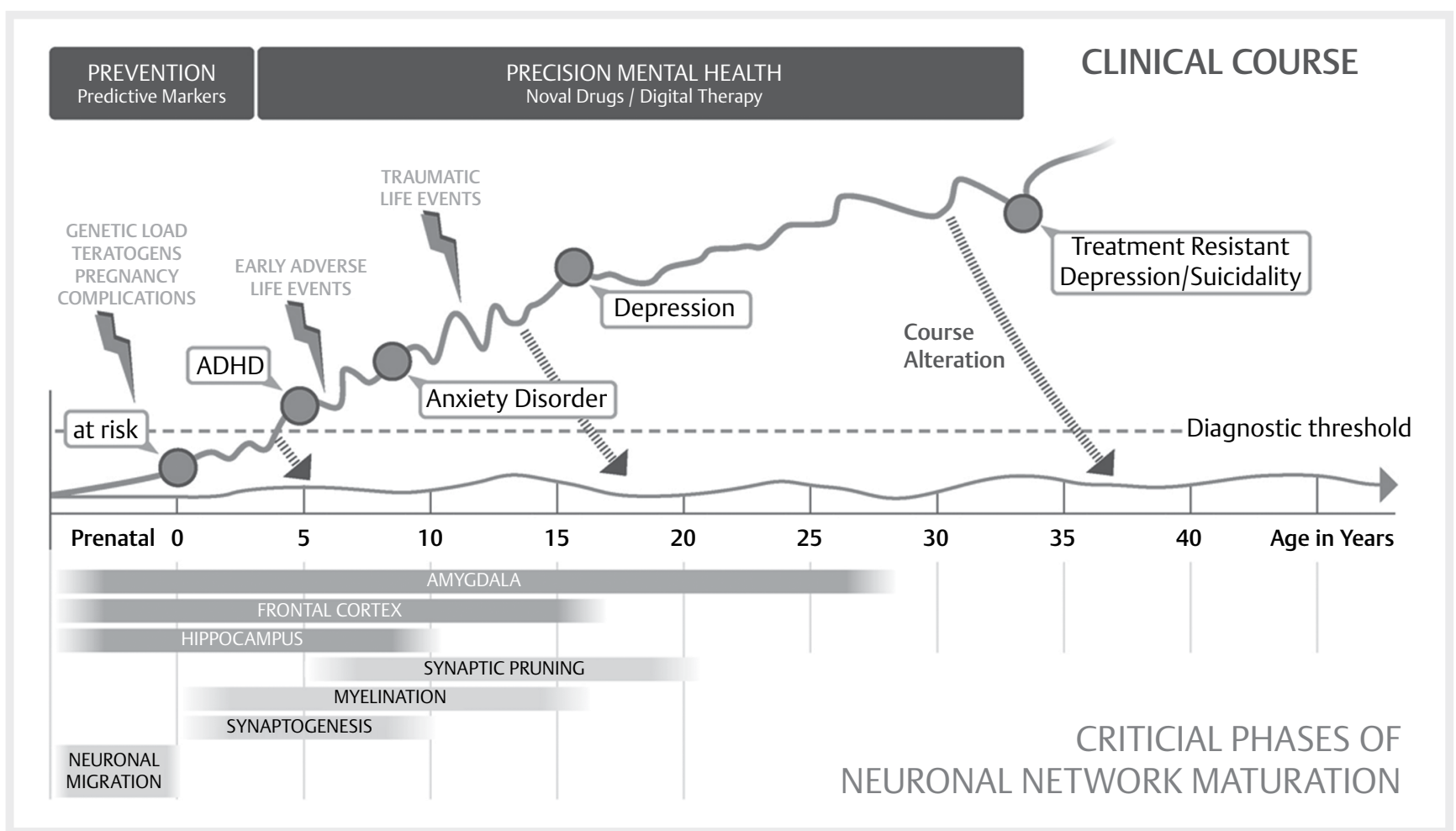

Fig. 2 Hypothetical trajectory of MDD over the lifespan. 


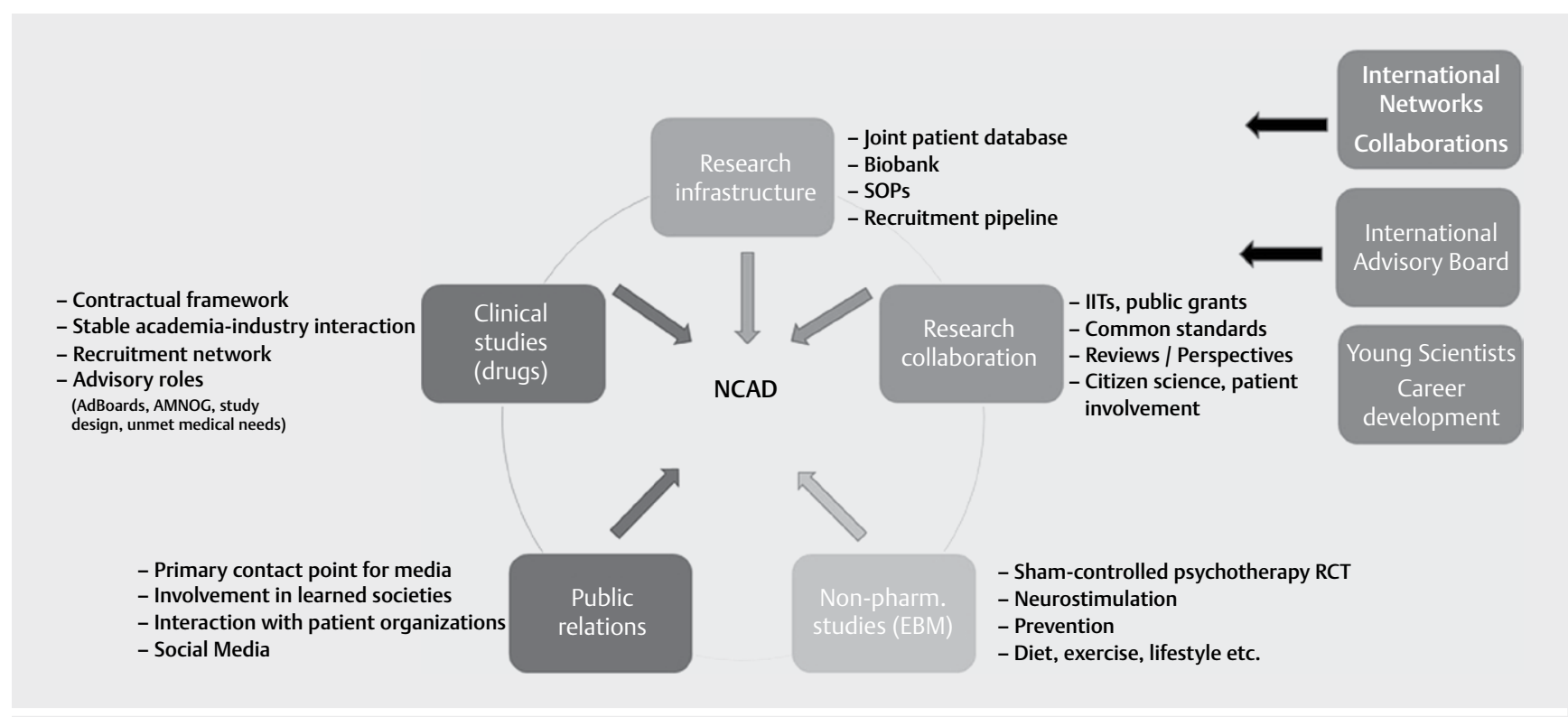

- Fig. 3 NCAD's structure and fields of work.

search units funded by the German Research Foundation (DFG) as well as international research networks, established within the Horizon 2020 framework of the European Commission.

The mission of the NCAD is to accomplish research in a highly translational manner, with structures that enable rapid conduct of translational clinical studies using academic as well as industry frameworks in a reliable way. To do so, the NCAD aims to extend existing and implement joint phenotyping protocols (including EMA approaches), biobanks with common standard operating procedures and a common recruitment pipeline. Coming along with that, the NCAD will set up a contractual template to speed up regulatory processes and legal negotiations, which are currently often time-consuming. In doing so, the NCAD shall be the major hub to conduct academic investigator-initiated trials (IIT), and the main partner to be approached by industry to conduct phase II, -III and -IV clinical studies in affective disorders. Importantly, studies are not limited to drug trials but also include well-controlled studies on neurostimulation and psychotherapy, where the lack of highlevel evidence from well-controlled trials is most evident. The NCAD comprises leading German institutions in the field of affective disorders. It is an excellent platform for translational approaches and effective recruitment for clinical studies through this unique cooperation.

Basic research at NCAD aims to identify the mechanisms driving the aetiology and development of affective disorders from school age to adulthood and their progression into chronic, severe stages of the disease. As mentioned above, currently applied diagnostic systems fall short in defining biological disease entities. NCAD will go beyond traditional classifications, thereby benefiting from extensive local experience in fundamental cognitive, affective, and social neuroscience research. In that way, NCAD strives to define mental disorders as a group of diseases that follow distinct trajectories in probabilistic ways. The integration of highly complex data (genetics and other omics approaches, neuroimaging, neu- ropsychology, deep behavioural phenotyping) using state-of-theart computational methods is essential to overcome un-mechanistic disease definitions imposed by current classifications.

However, the NCAD goes beyond being a clinical-translational research collaboration by also reaching out to the general public, patient organisations and learned societies. To do so, it closely collaborates e. g., with the German Depression Foundation (Stiftung Deutsche Depressionshilfe) or the German Society for Bipolar Disorders (Deutsche Gesellschaft für Bipolare Störungen, DGBS). The goal is to be the central contact point in Germany when it comes to translational studies in affective disorders in Germany ( $>$ Fig. $\mathbf{3}$ ). Also, NCAD will interact with international learned societies to disseminate findings and to integrate its research on the international level. Furthermore, the education of a new breed of clinical-translational clinician-scientists is also one of the core missions of NCAD.

Currently, the NCAD comprises eight academic centres with prominent expertise in affective disorders and their precursor disorders ADHD and ANX, namely the University Hospitals Bochum, Dresden, Erlangen, Frankfurt, Marburg, Münster, Regensburg, and Würzburg. The NCAD provides all clinical and research infrastructures ( $\vee$ Table 1 ) which are necessary to conduct basic research, as well as to recruit large numbers of patients suffering from affective disorders into PM and other clinical studies and trials both in an academic as well as the commercial framework. The NCAD is therefore Germany's main collaboration partner to conduct research on affective disorders and constitutes a multi-centre research framework which is urgently needed given the challenges outlined above.

\section{Acknowledgment}

NCAD partners have obtained joined grants from the Deutsche Forschungsgemeinschaft (DFG) (TRR SFB 58, FOR 2107, FOR 2858, GRK 2660, DFG GRK 2773/1), German Federal Ministry of Education and Research (BMBF)(BipoLife, OptiMD, ProtectAD, Panik- 


\begin{tabular}{|c|c|c|c|c|c|c|c|c|c|c|}
\hline & 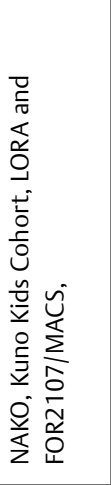 & 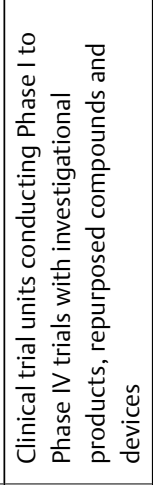 & 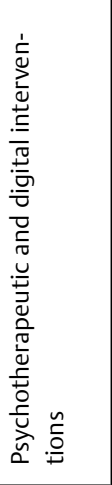 & 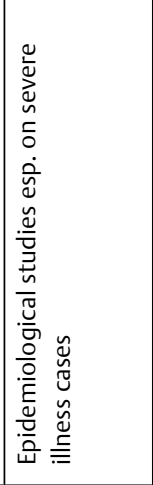 & 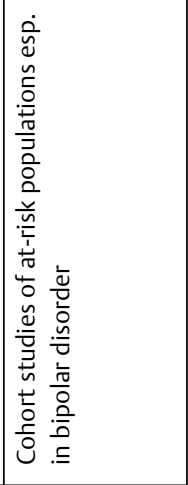 & 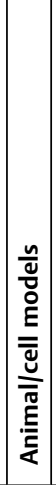 & 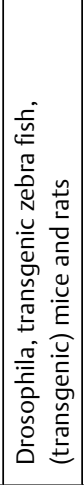 & 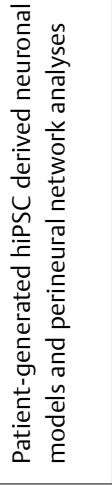 & & 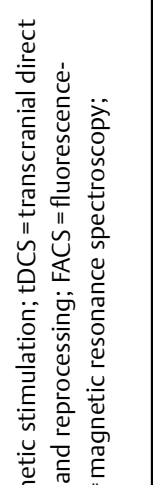 \\
\hline & $\frac{n}{0}$ & 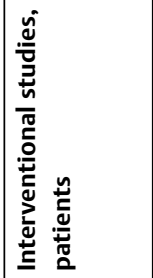 & 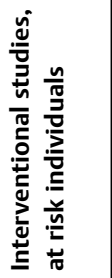 & 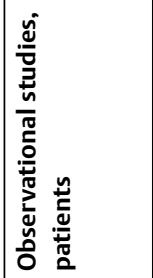 & 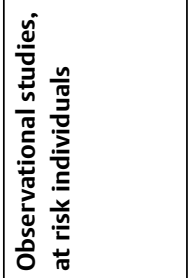 & & 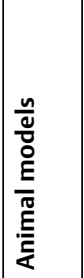 & $\begin{array}{l}\frac{n}{\bar{\Xi}} \\
\overline{0} \\
\underline{E} \\
\bar{\Xi}\end{array}$ & & 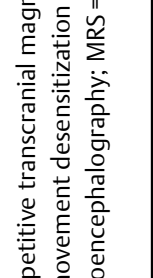 \\
\hline & 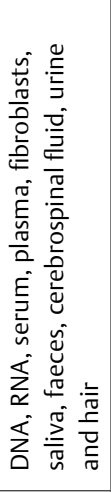 & 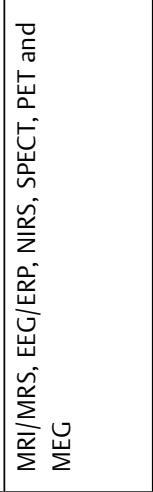 & 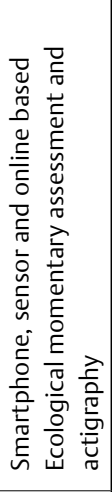 & 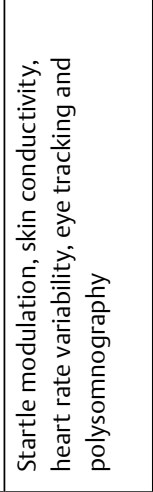 & 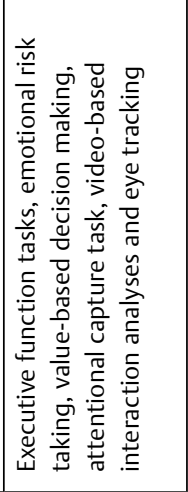 & 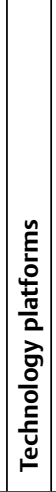 & 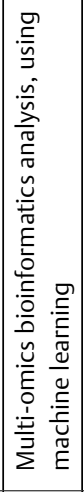 & 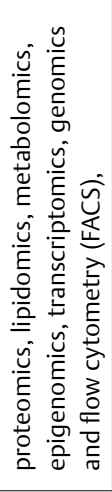 & & 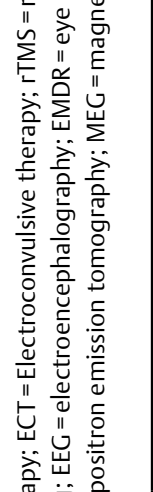 \\
\hline & 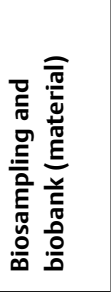 & 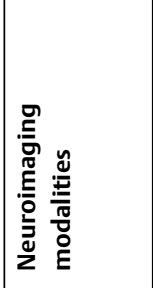 & 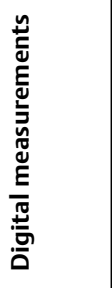 & 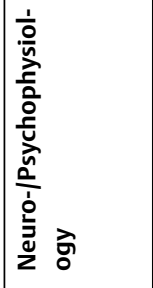 & 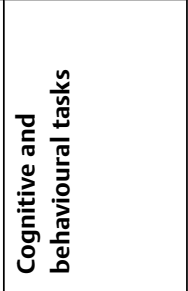 & & 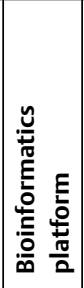 & 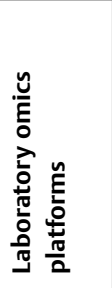 & & 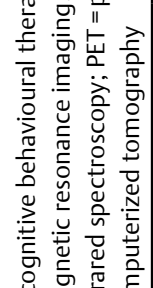 \\
\hline & 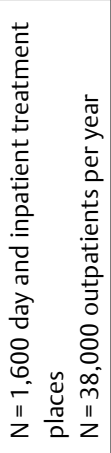 & 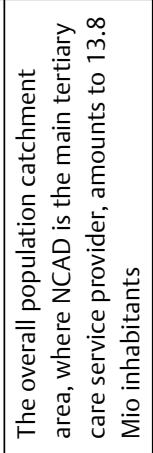 & 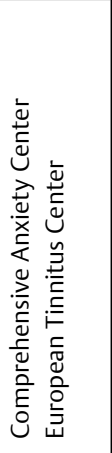 & 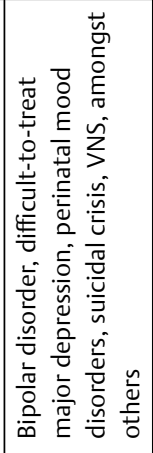 & 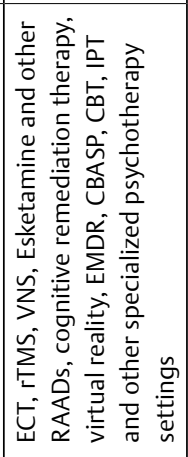 & & 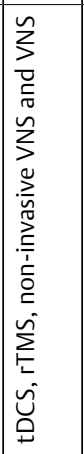 & 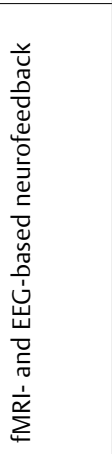 & 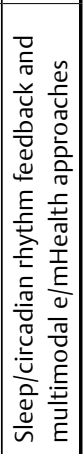 & 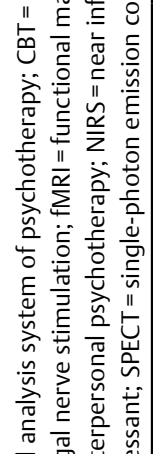 \\
\hline & 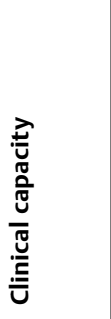 & 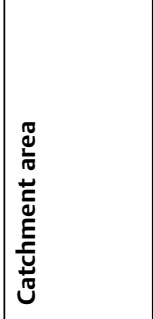 & 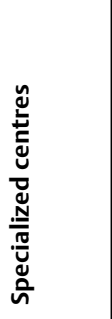 & 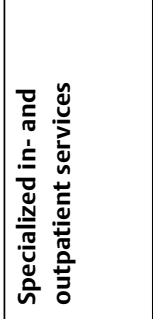 & 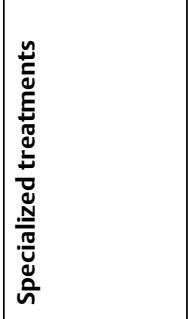 & 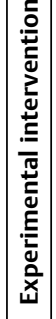 & 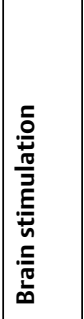 & 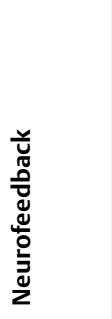 & 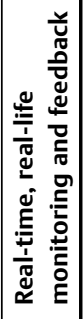 & 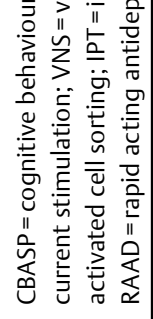 \\
\hline
\end{tabular}


Netz, earlyCBT, 01KG1307, EARLY), InnoFonds (CHIMPSNET, iCAN), and European Commission (grant agreements \#602805, 643051, 696802, 728018, 754907).

\section{Conflict of Interest}

The authors declare that they have no conflict of interest.

\section{References}

[1] Greenberg PE, Fournier AA, Sisitsky T, Pike CT, Kessler RC. The economic burden of adults with major depressive disorder in the United States (2005 and 2010). J Clin Psychiatry 2015; 76: 155-162

[2] Johnston KM, Powell LC, Anderson IM, Szabo S, Cline S. The burden of treatment-resistant depression: A systematic review of the economic and quality of life literature. J Affect Disord 2019; 242: 195-210

[3] Bessonova L, Ogden K, Doane M], O'Sullivan AK, Tohen M. The economic burden of bipolar disorder in the United States: A systematic literature review. Clinicoecon Outcomes Res 2020; 12: 481-497

[4] Wittchen HU, Jacobi F, Rehm J et al. The size and burden of mental disorders and other disorders of the brain in Europe 2010. Eur Neuropsychopharmacol 2011; 21: 655-679

[5] Konnopka A, Konig H. Economic burden of anxiety disorders: A systematic review and meta-analysis. Pharmacoeconomics 2020; 38: 25-37

[6] Angst J, Azorin JM, Bowden CL et al. Prevalence and characteristics of undiagnosed bipolar disorders in patients with a major depressive episode: The BRIDGE study. Arch Gen Psychiatry 2011; 68: 791-798

[7] Pelletier L, O'Donnell S, Dykxhoorn J, McRae L, Patten SB. Under-diagnosis of mood disorders in Canada. Epidemiol Psychiatr Sci 2017; 26 : 414-423

[8] Gogos A, Ney LJ, Seymour N, Van Rheenen TE, Felmingham KL. Sex differences in schizophrenia, bipolar disorder, and post-traumatic stress disorder: Are gonadal hormones the link? Br J Pharmacol 2019; 176: 4119-4135

[9] Hayes JF, Marston L, Walters K, King MB, Osborn DPJ. Mortality gap for people with bipolar disorder and schizophrenia: UK-based cohort study 2000-2014. Br J Psychiatry 2017; 211: 175-181

[10] Cuijpers P, Vogelzangs N, Twisk ], Kleiboer A, Li ], Penninx BW. Is excess mortality higher in depressed men than in depressed women? A meta-analytic comparison. J Affect Disord 2014; 161: 47-54

[11] Philpott LF, Savage E, FitzGerald S, Leahy-Warren P. Anxiety in fathers in the perinatal period: A systematic review. Midwifery 2019; 76: 54-101

[12] Edward KL, Castle D, Mills C, Davis L, Casey ]. An integrative review of paternal depression. Am J Mens Health 2015; 9: 26-34

[13] Bobo WV, Yawn BP. Concise review for physicians and other clinicians: Postpartum depression. Mayo Clin Proc 2014; 89: 835-844

[14] Schubert KO, Clark SR, Van LK, Collinson JL, Baune BT. Depressive symptom trajectories in late adolescence and early adulthood: A systematic review. Aust N Z J Psychiatry 2017; 51: 477-499

[15] Kaltenboeck A, Harmer C. The neuroscience of depressive disorders: A brief review of the past and some considerations about the future. Brain Neurosci Adv 2018; 2: 2398212818799269

[16] Kloiber S, Rosenblat JD, Husain MI et al. Neurodevelopmental pathways in bipolar disorder. Neurosci Biobehav Rev 2020; 112: 213-226

[17] Davidson JR. Major depressive disorder treatment guidelines in America and Europe. J Clin Psychiatry 2010; 71: e04
[18] Kishi T, Ikuta T, Matsuda Y et al. Mood stabilizers and/or antipsychotics for bipolar disorder in the maintenance phase: A systematic review and network meta-analysis of randomized controlled trials. Mol Psychiatry 2021; 26: 4146-4157

[19] Papakostas GI, Salloum NC, Hock RS et al. Efficacy of Esketamine augmentation in major depressive disorder: A meta-analysis. J Clin Psychiatry 2020; 81: 19r12889

[20] Galvao-Coelho NL, Marx W, Gonzalez M et al. Classic serotonergic psychedelics for mood and depressive symptoms: A meta-analysis of mood disorder patients and healthy participants. Psychopharmacology (Berl) 2021; 238: 341-354

[21] Razza LB, Palumbo P, Moffa AH et al. A systematic review and meta-analysis on the effects of transcranial direct current stimulation in depressive episodes. Depress Anxiety 2020; 37: 594-608

[22] Group UER. Efficacy and safety of electroconvulsive therapy in depressive disorders: A systematic review and meta-analysis. Lancet 2003; 361: 799-808

[23] Mutz J, Vipulananthan V, Carter B et al. Comparative efficacy and acceptability of non-surgical brain stimulation for the acute treatment of major depressive episodes in adults: Systematic review and network meta-analysis. BMJ 2019; 364: 11079

[24] Lovas DA, Schuman-Olivier Z. Mindfulness-based cognitive therapy for bipolar disorder: A systematic review. J Affect Disord 2018; 240: 247-261

[25] van Bronswijk S, Moopen N, Beijers L et al. Effectiveness of psychotherapy for treatment-resistant depression: a meta-analysis and meta-regression. Psychol Med 2019; 49: 366-379

[26] Konig IR, Fuchs $\mathrm{O}$, Hansen $\mathrm{G}$ et al. What is precision medicine? Eur Respir J 2017; 50: 1700391

[27] Lu YF, Goldstein DB, Angrist M et al. Personalized medicine and human genetic diversity. Cold Spring Harb Perspect Med 2014; 4: a008581

[28] Cuthbert BN, Insel TR. Toward new approaches to psychotic disorders: The NIMH Research Domain Criteria project. Schizophr Bull 2010; 36: 1061-1062

[29] Rosenblat JD, Kakar R, Berk M et al. Anti-inflammatory agents in the treatment of bipolar depression: a systematic review and meta-analysis. Bipolar Disord 2016; 18: 89-101

[30] Kohler-Forsberg O, NL C, Hjorthoj C et al. Efficacy of anti-inflammatory treatment on major depressive disorder or depressive symptoms: Meta-analysis of clinical trials. Acta Psychiatr Scand 2019; 139: 404-419

[31] Ludwig B, Dwivedi Y. Dissecting bipolar disorder complexity through epigenomic approach. Mol Psychiatry 2016; 21: 1490-1498

[32] Matosin N, Halldorsdottir T, Binder EB. Understanding the molecular mechanisms underpinning gene by environment interactions in psychiatric disorders: The FKBP5 model. Biol Psychiatry 2018; 83: 821-830

[33] Weintraub M], Schneck CD, Axelson DA et al. Classifying mood symptom trajectories in adolescents with bipolar disorder. J Am Acad Child Adolesc Psychiatry 2020; 59: 381-390

[34] Scott K, Lewis CC, Marti CN. Trajectories of symptom change in the treatment for adolescents with depression study. J Am Acad Child Adolesc Psychiatry 2019; 58: 319-328

[35] Shaw P, Sudre G. Adolescent attention-deficit/hyperactivity disorder: Understanding teenage symptom trajectories. Biol Psychiatry 2021; 89: 152-161

[36] Faedda GL, Serra G, Marangoni C et al. Clinical risk factors for bipolar disorders: A systematic review of prospective studies. J Affect Disord 2014; 168: 314-321

[37] Franke B, Michelini G, Asherson P et al. Live fast, die young? A review on the developmental trajectories of ADHD across the lifespan. Eur Neuropsychopharmacol 2018; 28: 1059-1088 
[38] Remes O, Brayne C, van der Linde R et al. A systematic review of reviews on the prevalence of anxiety disorders in adult populations. Brain Behav 2016; 6: e00497

[39] Gross-Lesch S, Dempfle A, Reichert S et al. Sex- and subtype-related differences in the comorbidity of adult ADHDs. J Atten Disord 2016; 20: 866

[40] Melton TH, Croarkin PE, Strawn JR et al. Comorbid anxiety and depressive symptoms in children and adolescents: A systematic review and analysis. J Psychiatr Pract 2016; 22: 84-98

[41] de la Fuente-Tomas L, Sierra P, Sanchez-Autet M et al. A clinical staging model for bipolar disorder: longitudinal approach. Transl Psychiatry 2020; 10: 45

[42] Vieta E, Salagre E, Grande I et al. Early intervention in bipolar disorder. Am J Psychiatry 2018; 175: 411-426

[43] Davey CG, McGorry PD. Early intervention for depression in young people: A blind spot in mental health care. Lancet Psychiatry 2019; 6: 267-272

[44] Zhang Z, Zhang L, Zhang G et al. The effect of CBT and its modifications for relapse prevention in major depressive disorder: A systematic review and meta-analysis. BMC Psychiatry 2018; 18: 50

[45] Lindner P, Hamilton W, Miloff A et al. How to treat depression with low-intensity virtual reality Interventions: Perspectives on translating cognitive behavioral techniques into the virtual reality modality and how to make anti-depressive use of virtual reality-unique eExperiences. Front Psychiatry 2019; 10: 792

[46] Fodor LA, Cotet CD, Cuijpers $P$ et al. The effectiveness of virtual reality based interventions for symptoms of anxiety and depression: A meta-analysis. Sci Rep 2018; 8: 10323
[47] Jahn FS, Skovbye M, Obenhausen K et al. Cognitive training with fully immersive virtual reality in patients with neurological and psychiatric disorders: A systematic review of randomized controlled trials. Psychiatry Res 2021; 300: 113928

[48] Horlyck LD, Obenhausen K, Jansari A et al. Virtual reality assessment of daily life executive functions in mood disorders: associations with neuropsychological and functional measures. J Affect Disord 2021; 280: 478-487

[49] Pfennig A, Leopold K, Martini J et al. Improving early recognition and intervention in people at increased risk for the development of bipolar disorder: Study protocol of a prospective-longitudinal, naturalistic cohort study (Early-BipoLife). Int J Bipolar Disord 2020; 8: 22

[50] Ritter PS, Bermpohl F, Gruber O et al. Aims and structure of the German Research Consortium BipoLife for the study of bipolar disorder. Int J Bipolar Disord 2016; 4: 26

[51] Muhlbauer E, Bauer M, Ebner-Priemer U et al. Effectiveness of smartphone-based ambulatory assessment (SBAA-BD) including a predicting system for upcoming episodes in the long-term treatment of patients with bipolar disorders: Study protocol for a randomized controlled single-blind trial. BMC Psychiatry 2018; 18: 349

[52] Bauer M, Banaschewski T, Heinz A et al. [The German research network for mental disorders]. Nervenarzt 2016; 87: 989-1010

[53] Kircher T, Wohr M, Nenadic I et al. Neurobiology of the major psychoses: A translational perspective on brain structure and function-the FOR2107 consortium. Eur Arch Psychiatry Clin Neurosci 2019; 269: 949-962 